\title{
TYPE II BURSTS OF DIFFERENT SUBCLASSES ASSOCIATED TO TRANSIENTS AND MASS EJECTION IN THE HIGHER CORONA
}

\author{
H.W. URBARZ \\ Dataarchives of the Astronomical Institute of the University of Tuebingen \\ Weissenau Station, Rasthalde, D-88214 Ravensburg Germany \\ Fax+49-7544-71306, Email INET:100331.1131@compuserve.com
}

December 1, 1996

\begin{abstract}
The list of mass ejections published in Solar Geophysical Data during the period of Jan. 1981 to Oct. 1987 contains about 1300 Surges, Sprays and Type II bursts. The relationship between the mass ejection events and the shock front events is investigated by means of correlation of time and position. The result shows that type II bursts start as frequently after the mass ejections as prior to them.
\end{abstract}

\section{Introduction}

Mass ejections of different kinds observed by ground based instruments or observed by satellite borne instruments have been investigated regarding flare related events whose velocities are faster by the factor of 1.67 than the associated transients impling that the shocks were piston driven. The number of samples were only 27 but they had a broad rms dispersion of velocity and all were related to optical transients. But from a group of transients only a fraction is related to type II bursts (Gergely 1984). A plot of 10 optically observed transients showing height versus speed was published by Hildner (1977). The maximum speeds were $700 \mathrm{~km} / \mathrm{s}$, with only one having a somewhat higher speed. It is known from a number of observations of associated events that the related type II bursts may travel faster by a factor of 3 than the optical transient. By means of radioheliographic observations, the relationship of CME and type II bursts was investigated regarding timing, relative location and relative velocity (Gopalsvamy 1990). There was one case of a rather slow CME and a fast shock that had a different origins. In a different case a CME moving slowly outward was followed by a fast shock front overtaking it. In both cases a relation of cause and effect was not indicated. The last two samples showed also that relations between associated events may turn out to be rather complex, if sensitive and high resolution instruments are used. The conventional image of type II related shock fronts driven by mass ejection was postulated again by Gurnett (1995) which was a rather far reaching generalisation. 


\section{A List of Surges, Sprays, Type II Bursts}

A large number of events of the kind mentioned is available in the monthly editions of the Solar Geophysical Data II during January 1, 1980 to October 16,1987 . We confine our investigation to this interval of time and refer only to surges, sprays and type II bursts. The following lines describe a sample of events in the list, ie. February 07, 1986 and of February 22, 1985.

TABLE I

Mass Ejection from the Sun

\begin{tabular}{lllllll}
\hline Date & Start & $\begin{array}{l}\text { End } \\
\text { Max }\end{array}$ & \multicolumn{2}{c}{ Location } & type of & Dt \\
RAo & R/Ro & event & UT \\
\hline Febr. 07. 86 & 1011.5 & 1027.0 & $\mathrm{dm}$ & $\mathrm{mII}$ & $+0.33 .5 \mathrm{~min}$ & \\
Febr. 07. 86 & 1035 & $1142 \mathrm{D}$ & 258 & 0.40 & Sp & \\
Febr. 07. 85 & $1011 \mathrm{E}$ & $1031 \mathrm{D}$ & 076 & 0.75 & S 1015 & $-2.37 \mathrm{~h}$ \\
Febr. 22. 85 & 1248.3 & 1258 & & II Harm. & HB & \\
\hline
\end{tabular}

\section{Legend:}

$\mathrm{Sp}=$ spray $\mathrm{S}=$ surge (observed in Halpha) $\mathrm{dm}, \mathrm{m} \mathrm{II}=$ decametric, metric type II burst Harm, $\mathrm{HB}=$ harmonic band, resp. herringbones

$\mathrm{Dt}=$ starttime of $\mathrm{S}$ or $\mathrm{Sp}$ minus starttime of type II, this correlation was done for values of $\mathrm{Dt}$ up to $\pm 3 \mathrm{~h}$.

$\mathrm{RAo}=$ angle of radius vector $\mathrm{R} / \mathrm{Ro}=$ lengths of radius vector, both values measured on the solar disc, see Explanations for mass ejection, Supplement 1987 and Solar Geophysical Data II, 601, Sept. 1994. The listing was terminated in March 1994.

Different observatories were contributing ground based coronagraph data during the interval in question, so the data material is not homogeneous. Flares, sprays and flare surges were listed only if the associated flares was known. These events and type II bursts from moving type IV burst from this list are considered in our correlation; other events like moving type IV bursts, prominences were not considered.

\section{Statistics of Surges, Sprays and Type II Bursts}

The table 2 gives a survey of the events contained in the list presented in Section 2. 
TABLE II

\begin{tabular}{|l|l|}
\hline Total number of Surges & $\mathrm{n}=1134$ \\
Total number of Sprays & $\mathrm{n}=166$ \\
Total number of Type II bursts & $\mathrm{n}=406$ \\
Total number of correlated events & \\
in the sense given in Section 2 & $\mathrm{n}=44$ \\
Total of correlated Surges - Type II & $\mathrm{n}=24: 2.12 \%$ \\
Total of correlated Sprays - Type II & $\mathrm{n}=20: 1.76 \%$ \\
\hline
\end{tabular}

We notice that of all the possible Surges $35.7 \%$ and $14.6 \%$ were associated with sprays and Type II bursts respectively. The question whether the data is complete is not very important. The number of type II bursts seems to be nearly correct compared to other listings. The problem is to explain the low correlation presented in Table 2, because as found before the correlation of type II bursts with transients is almost $100 \%$. Moreover the H-alpha events considered here and type II bursts are known to be due to flares. Thus we suggest that both types of $\mathrm{H}$-alpha events represent phenomena that are different from the transients observed by satellite bound coronographs. These form dense extended clouds of magnetized plasma in contrast to the compact clouds of small diameter originating at lower levels and being incapable of forming shock fronts. Transients are produced by extending loops. In Fig. 1 we see the distribution over the relevant period of the number of uncorrelated surges and type II bursts. The number of the type II events decreases faster toward the year of Solar Minimum near 1985 than the number of surges which however show a seasonal distribution with a drastic increase during each summer.

\section{What comes first, the Shock Front or the Mass Ejection?}

In the list of section 2 the position of the $\mathrm{H}$-alpha events projected on the solar disc is given together with the start times of both the $\mathrm{H}$-alpha events and the type II bursts. We assume that correlated events do occur at some level along the radius vector. If the start time of a type II burst occurs prior to the $\mathrm{H}$-alpha event, the shock front is located ahead of the surge or spray and vice versa. In Fig. 2, the time differences given by the values $\mathrm{Dt}$ are plotted separately for the surges and the sprays in arbitrary intervals. We find that the $\mathrm{H}$-alpha events may move as often ahead of the shock front as behind it. Thus the picture of a driven shock front always holds. In order to test these results, the type II data should be compared with those of Solar 


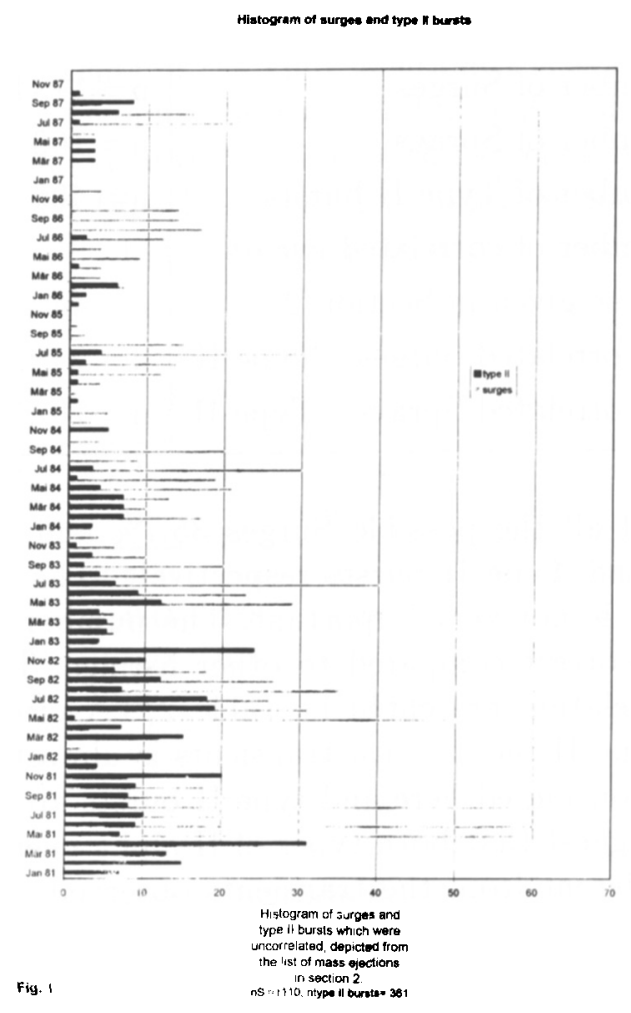

Fig. 1.

Geophysical Data and of UAG Rep.98. Moreover the positions of the active regions giving rise to the type II bursts and the coronal heights derived from the start frequencies may be compared with the data of the radius vector.

\section{Acknowledgements}

Thanks are due to the Organizers of the 154 IAU Colloquim at NCRA, Pune, for accepting this contribution.

\section{References}

T.E. Gergely, 1984, 'The relative velocity of coronal transients and type II bursts', STIP Symposium on Interplanetary Intervals, Proc. of the Maynooth Symp. held in Aug. 1982, ed. M.A. Shea and D.F. Smart and S.M.P. McKenna-Lawlor, 347-357

E. Hildner, 1977, 'Mass ejection from the solar corona into interplanerary space' Study of Traveling Interplanery Phenomena, Proceedings of the L.D.De Feiter Memorial 


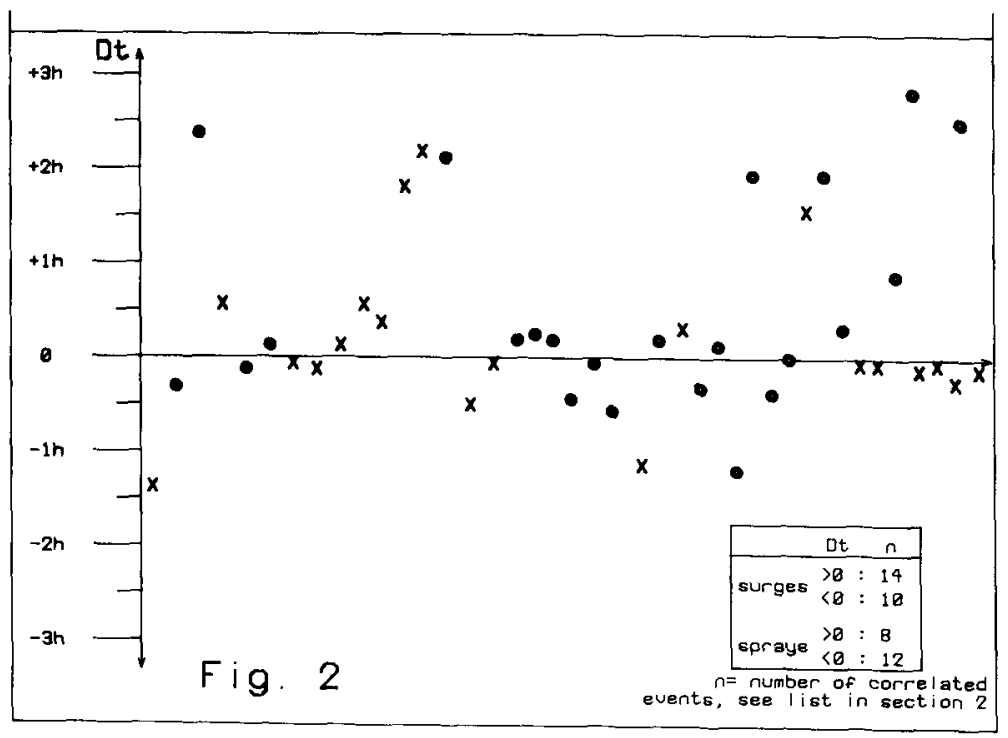

Fig. 2.

Symposium held in Tel Aviv, June 1977, Ed.M.A. Shea and D.F. Smart and S.T. Wu, 3-22

N. Gopalswamy, 1980, 'Clark Lake Radio Observations of the Coronal Mass Ejection', Basic Plasma Processes on the Sun, Proceedings of the 142th Symposium of the IAU held in Bangalore, Dec. 1989, ed. by E.R. Priest and V. Krishnan, 495-500

D.A. Gurnett, 1995, 'Heliospheric Radio Emission', The High Latitude Heliosphere, Proceedings of the 28th ESLAB Symposium, April, 1994, Friedrichshafen, ed. R.G. Marsden, 243-254

H.W. Urbarz, 1990, 'Type II Solar Radio Bursts recorded at Weissenau 1966- 1987', World Data Center A for Solar-Terrestrial Physics Report UAG-98, Boulder Col. A.SD. A. Febr. 82 pages 